\title{
Desarrollo Teórico de la Energía de Excitones en Microcavidades Semiconductoras de Capas Delgadas Generadas en Materiales de ZnSe y CdSe
}

\section{Theoretical Development of Exciton Energy in Semiconductor Microcavities of Thin Layers Generated in $\mathrm{ZnSe}$ and CdSe Materials}

\author{
C. Marroquín ${ }^{1}$ | A. EucedA ${ }^{2}$
}

Recibido: 2 de febrero de 2019/ Aceptado: 1 de diciembre de 2019

\begin{abstract}
Se modela la energía del estado base para el electrón y hueco confinados en un pozo cuántico de paredes delgadas y altura de potencial finita, en una estructura semiconductora de $\mathrm{ZnSe} / \mathrm{CdSe} / \mathrm{ZnSe}$, mediante el desarrollo de la ecuación transcendental, a partir de las ecuaciones de onda. Se presenta la energía de enlace excitónica $E_{b}$ y la energía de fotoluminiscencia FL en función del ancho de pozo $L$ mediante las deposiciones de capaz delgadas de CdSe sobre el ZnSe en la dirección $z$, utilizando la aproximación del método variacional y de masa efectiva, cada uno de los resultados aquí obtenidos mostraron una buena aproximación con los reportados en investigaciones experimentales.
\end{abstract}

The base state energy for the electron and hollow confined in a thin-walled quantum well and finite potential height in a semiconductor structure of $\mathrm{ZnSe} / \mathrm{CdSe} / \mathrm{ZnSe}$ is modeled by the development of the transcendental equation, from equations cool. The excitonic bond energy $E_{b}$ and the photoluminescence energy FL as a function of the wellbore $L$ are presented by the capacitance depositions of CdSe thin on the ZnSe in the $z$ direction, using the variational method and effective mass approach, each Of the results obtained here showed a good approximation with those reported in experimental investigations.

\section{PALABRAS CLAVES}

Ecuación Trascendental; Pozo Cuántico; Excitón; Fotoluminiscencia

\section{KEYWORDS}

Transcendental Equation; Quantum Well; Exciton; Photoluminescence.

PACS

81.05.Hd
${ }^{1}$ Escuela de Física, Facultad de Ciencias, Universidad Nacional Autónoma de Honduras email: carlos.marroquin@unah.edu.hn

${ }^{2}$ Escuela de Física, Facultad de Ciencias, Universidad Nacional Autónoma de Honduras email: aeunah@gmail.com 


\section{I | INTRODUCCIÓN}

$\mathrm{E}^{\mathrm{N}}$ el siglo XXI se están desarrollado avances importantes en la ciencia y la tecnología de los materiales que se ligan estrechamente, diversos experimentos con materiales semiconductores han permitido diseñar y preparar multicapas semiconductoras, para formar heteroestructuras de dimensiones nanométricas, haciendo deposiciones capa por capa atómica de un material sobre otro, denominado este último sustrato, lográndose este evento con la utilización de diferentes técnicas de crecimiento.

La transmisión de la tecnología y de la información de manera eficiente se logra cuando se estudian materiales cuyas características tengan propiedades fisicoquímicas que favorezcan el diseño y construcción de dispositivos de baja dimensión, como ser diodos láser, transistores, sensores opto electrónicos, fotodetectores entre otros, que es común en nuestro medio pasen desapercibidos cuando se utiliza el teléfono celular, el control remoto para encender la televisión y la televisión misma etc.

El estudio de los materiales semiconductores del grupo II-VI que se localizan en la tabla periódica y de manera específica en los compuestos $\mathrm{ZnSe}$ y CdSe, han sido motivo de estudio y utilizados para la elaboración de diodos emisores de luz presentando un rango de espectro visible y con emisiones de luz entre el azul y verde. (P. Diaz Arencibia, 2014) (A. Escamilla Esquivel, 1997)

Otra de las características de estos semiconductores es que presentan una energía de brecha prohibida directa $E_{g}$, dicho acople de estos semiconductores (ZnSe y CdSe) presentan distinta energía prohibidas (Bandgap), por ejemplo la brecha de energía del compuesto ZnSe es mayor que la brecha de energía del compuesto CdSe, este último semiconductor se deposita sobre el primero, dando lugar a la formación de heteroestructuras tipo I, llamada pozo cuántico simple, lo que permite confinar y controlar tanto el electrón como el hueco en una misma región del espacio, el sistema así formado se denomina exciton, que tiene similitud al átomo de hidrogeno (hidrogenoide)(A. Londoño Calderon, 2011).

Se utiliza un método gráfico para desarrollar la ecuación transcendental, que permite calcular la energía de estado base del electrón y hueco respectivamente, Se desarrolla de forma aproximada la ecuación de Schrödinger para este tipo de heteroestructura de paredes finitas, utilizando el principio variacional y la aproximación de masa efectiva, que posteriormente se utiliza para el cálculo de la energía de enlace excitonico y energía de fotoluminiscencia, para contrastar estos cálculos teóricos se modela mediante una gráfica la longitud de onda en función de la energía emitida por el electrón (recombinación).(Robert Eisberg, 2009)(Chuang, 1995)(Grundmann, 2010)

Se modela la energía de enlace de un exciton en función del ancho del pozo cuántico, que se genera cuando un electrón localizado en la banda de valencia absorbe energía y salta a un nivel excitado, de igual forma la energía de transición energética en función del ancho del pozo, analizando en el gráfico la longitud de onda en función de la energía de fotoluminiscencia.

\section{II | MARCO TEÓRICO}

Aplicando el método variacional el cual permite definir una función de prueba dado por $\varphi\left(\rho, z_{e}, z_{h}\right)$ y escrita de la forma:

$$
\varphi\left(\rho, z_{e}, z_{h}\right)=N f_{e}\left(z_{e}\right) f_{h}\left(z_{h}\right) g\left(\rho, z_{e}, z_{h}\right)
$$




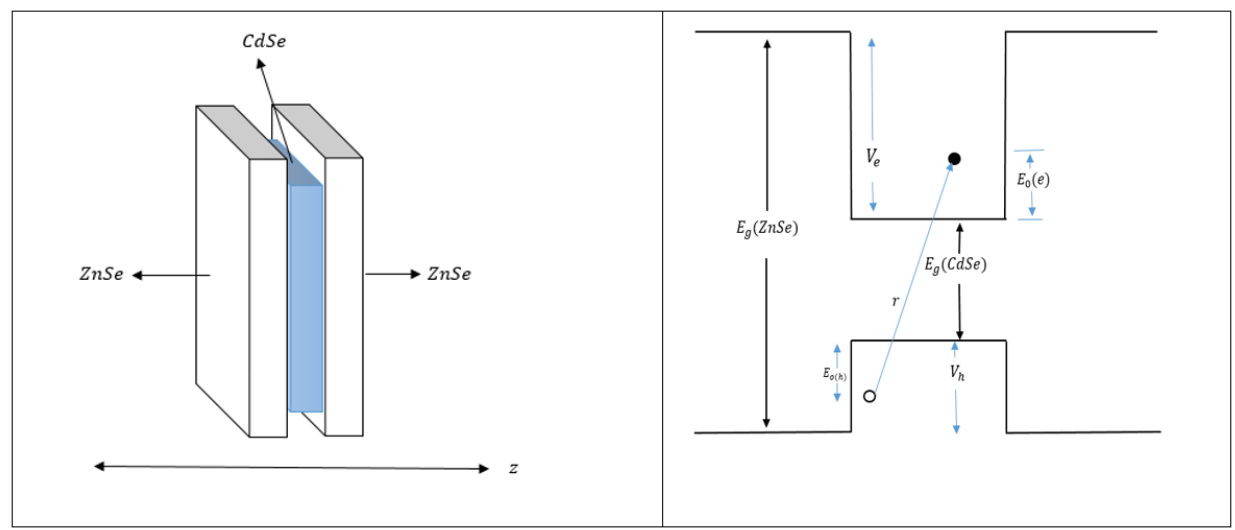

Figura 1: Estructura de bandas para el pozo cuántico de ZnSe/CdSe/ZnSe.

Siendo $N$ una constante de normalización, $f_{e}$ y $f_{h}$ son las soluciones para los estados base de electrón y hueco respectivamente en un pozo cuántico de paredes finitas (P. Aristizábal (2007)).

Donde la solución del electrón $f_{e}$ tiene la forma:

$$
\begin{gathered}
f_{e I}=\left[\cos \left(\frac{k_{e} L}{2}\right) e^{\alpha \frac{L}{2}}\right] e^{\alpha z_{e}} ; \quad z_{e} \leq-\frac{L}{2} \\
f_{e I I}=\cos k_{e} z_{e} ;-\frac{L}{2} \leq z_{e} \leq \frac{L}{2} \\
f_{e I I I}=\left[\cos \left(\frac{k_{e} L}{2}\right) e^{\alpha \frac{L}{2}}\right] e^{-\alpha z_{e}} ; z_{e} \geq \frac{L}{2}
\end{gathered}
$$

Donde $L$ es el ancho del pozo, $\alpha_{e}=\sqrt{\frac{2 m_{e} *\left(V_{e}-E_{e}\right)}{\hbar^{2}}}$ y $k_{e}=\sqrt{\frac{2 m_{e} * E_{e}}{\hbar^{2}}}, m_{e}^{*}$ es la masa efectiva del electrón, $V_{e}$ es el potencial de confinamiento en función del ancho del pozo $L$ y $E_{e}$ es la energía del estado base del electrón. De manera similar la función $f_{h}$ para hueco se define con los parámetros que corresponden al mismo. Para el cálculo del potencial de confinamiento se consideró $75 \% \Delta E_{g}$ para el electrón y $25 \% \Delta E_{g}$ para el hueco, siendo $\Delta E_{g}=E_{g}(\mathrm{ZnSe})-E_{g}(\mathrm{CdSe})$ como se observa la figura 1 (A. Londoño Calderon, 2011)(Robert Eisberg, 2009).

Para obtener los valores de la energía del estado base para el electrón y el hueco, considerando las condiciones de frontera en las tres regiones del pozo cuántico la ecuación transcendental tiene la forma:

$$
\left(\frac{2 m_{e}^{*} E_{e} L^{2}}{\hbar^{2}}\right)^{1 / 2} \tan \left(\frac{k_{e} L}{2}\right)=\sqrt{\frac{2 m_{e}^{*}\left(V_{e}-E_{e}\right) L^{2}}{\hbar^{2}}}
$$

Se utilizo un método gráfico para desarrollar la ecuación trascendental, tomando diferentes valores para el ancho del pozo cuántico $L$. En el caso de la función $g\left(\rho, z_{e}, z_{h}\right)$ expresa el estado de los átomos tipo hidrogenoide $1 s$, y tiene la forma: $g\left(\rho, z_{e}, z_{h}\right)=e^{(-\lambda r)}$ (Chaudhuri, 1984), donde $r=\sqrt{\left(\rho^{2}+\left(z_{e}-z_{h}\right)^{2}\right)}$ es la distancia entre el electrón y el hueco (figura 1) y $\lambda$ es el parámetro variacional que se obtiene a 
partir del calculo de la energía mínima.

Para determinar la energía de enlace excitonica del Sistema se utiliza la expresión.

$$
E_{b}=E_{e}+E_{h}-\langle\psi|H| \psi\rangle_{\text {min }}
$$

Donde $\langle\psi|H| \psi\rangle_{\min }$ es la energía minima del par electron-hueco y $H$ es el hamiltoniano para un exciton el cual se expresa por:

$$
H=-\frac{h^{2}}{2 \mu}\left[\frac{1}{\rho} \frac{\partial}{\partial \rho}\left(\rho \frac{\partial \partial}{\partial \rho}\right)+\frac{1}{\rho^{2}} \frac{\partial^{2}}{\partial \phi^{2}}\right]-\frac{h^{2}}{2 m_{e}} \frac{\partial^{2}}{\partial z_{e}^{2}}-\frac{h^{2}}{2 m_{h}} \frac{\partial^{2}}{\partial z_{h}^{2}}-\frac{e^{2}}{\varepsilon r}+V_{e}\left(z_{e}\right)+V_{h}\left(z_{h}\right)
$$

$m_{e}^{*}$ y $m_{h}^{*}$ son la masa efectiva del electrón y hueco respectivamente, $\varepsilon$ es la constante dieléctrica para $\mathrm{CdSe}, \mu$ es la masa reducida del Sistema localizada perpendicularmente al crecimiento de las capaz de material de la dirección $z$ (R. L. Greene, 1984).

Para determinar la energía de fotoluminiscencia FL del sistema se utiliza la expresión.

$$
E_{F L}=E_{e}+E_{h}+E_{g}-E_{b}
$$

\section{III | ANÁLISIS DE RESULTADOS}

Resolviendo la ecuación trascendental (5) para diferentes valores del ancho de pozo $L$ se obtienen la variación de la energía del estado base del electrón con un comportamiento tipo parabólico, como muestra la figura (2).

La gráfica de la figura 2, muestra que al variar el ancho del pozo en el intervalo de $0 \leq L \leq 16$ se observa dos situaciones, primero, a medida que el ancho del pozo L se acerca a cero la energía del estado base del electrón tiende a un valor máximo aproximado de $0.8 \mathrm{eV}$, y en donde la función de onda coincide con el valor máximo del potencial del electrón $V_{e}\left(z_{e}\right)$ que se localiza en la banda de conducción del material ZnSe. Segunda situación, si el ancho del pozo aumenta, la probabilidad de confinamiento del electrón es baja, en consecuencia la energía tiende a cero, y la función de onda coincidiendo con la banda de valencia del material CdSe en el pozo cuántico.

De igual forma para obtener la energía del estado fundamental del hueco, cuando el ancho del pozo tiende a cero su valor energético es de $0.26 \mathrm{eV}$ y con un valor de cero cuando $L$ tiende al infinito, como se muestra en la figura (3).

La variación de la energía de enlace $\left(E_{b}\right)$ del exciton en el pozo cuántico de ZnSe/CdSe/ZnSe, como una función del ancho $(L)$, se logra a partir del desarrollo de la ecuación (6), la figura (4) describe la variación de la energía de enlace del exciton y en ella se observa que a medida que el ancho del pozo $L$ crece la energía del sistema excitonico tiende a un valor mínimo constante de $16 \mathrm{meV}$, y con una tendencia muy alta de que el exciton se localice fuera de la región de confinamiento, este efecto se debe a que en la interface de la heteroestructura de $\mathrm{ZnSe} / \mathrm{CdSe}$ se produce un relajamiento y una dislocación, debido a que el $\mathrm{ZnSe}$ y CdSe presentan diferentes constantes de red, es decir 5.676 ̊ y $6.052 \AA$ A respectivamente (T. Yao, 1998).

Por otro lado en el intervalo de 0 a $10 \AA$ se observa que existe una máxima energía de enlace de 


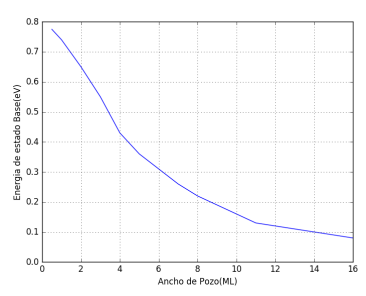

Figura 2: Variación de la energía del estado base del electrón en función del ancho del pozo en monocapa.

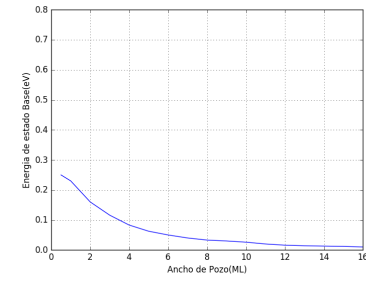

Figura 3: Variación de la energía del estado base del hueco en función del ancho del pozo en monocapa.

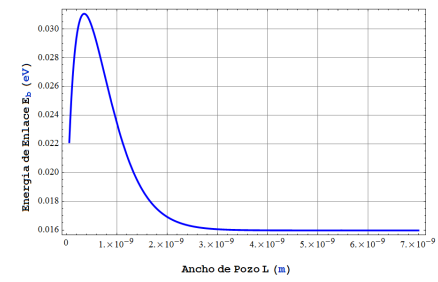

Figura 4: Variación de la energía de enlace del exciton

$31.5 \mathrm{meV}$, cuando el ancho del pozo $L \approx 3.8 \AA$, que se aproxima al espesor critico $(34.13 \mathrm{meV})$ reportado (D. Litvinovl (2008a)), lo que representa una diferencia de $2.63 \mathrm{meV}$. La energía de enlace aproximada de $21 \mathrm{meV}$ se obtiene cuando $L$ tiende a 0 , por lo que existe la probabilidad de encontrar el exciton en la región de confinamiento.

La figura (5a) muestra que la energía de fotoluminiscencia decrece cuando el ancho del pozo tiende a su valor crítico, de $3.8 \AA$. una vez que el portador de carga(electrón) que se localiza en la banda de conducción recombina hacia un hueco dejado durante el proceso de absorción en la banda de valencia, emitiendo luz con una energía $E=h c / \lambda$, asociada a esta energía hay una longitud de onda, a la cual le corresponde un color del espectro visible.

Es importante mencionar de acuerdo a la ecuación (7) que la energía de enlace en este tipo de estructura ( $\mathrm{ZnSe} / \mathrm{CdSe} / \mathrm{ZnSe}$ ) juega un papel muy importante en la intensidad de la emisión radiactiva al ocurrir el efecto de fotoluminiscencia, es decir, en la medida que la energía de enlace del exciton aumenta en función del ancho de pozo, la probabilidad de encontrar al exciton en la región de confinamiento es mayor como se muestra en la figura (5).

Al compararla los resultados con los datos experimentales reportados en (D. Litvinovl, 2008b)(V. Pellegrini, 1995)(I. Hernández-Calderón, 2005) muestran una buena aproximación. Para identificar el espectro de emisión de este material (CdSe) se calculó la longitud de onda en función de la energía de fotoluminiscencia $\left(E_{F L}\right)$ mediante la ecuación básica de espectroscopía como se mencionó anteriormente $E=h c / \lambda$, como se muestra en la figura $(5 \mathrm{~b})$.

A continuación (ver tabla 1) se presenta un cuadro comparativo con los resultados teóricos obtenidos y los experimentales.

\section{IV | CONCLUSIÓN}

Este trabajo de investigación se centró en tres aspectos fundamentales, el cálculo numérico de la energía de enlace, la energía de transición electrónica o fotoluminiscencia (FL) y la comparación de los resultados teórico numérico aquí calculados con los resultados experimentales obtenidos de reportes de varios autores. Se resolvió gráficamente la ecuación trascendental, utilizando para ello wolfram mathematica 7, el cual es un programa amigable, con el fin de obtener la energía del estado fundamental 


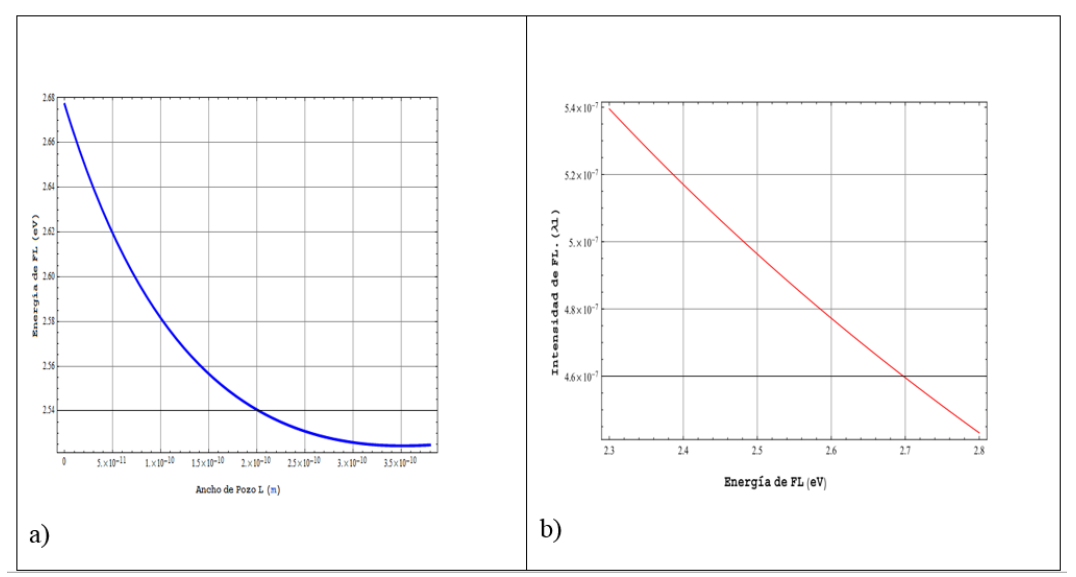

Figura 5: Variación de la energía de transición excitonica, en función del ancho del pozo cuántico y la longitud de onda en función de la energía de fotoluminiscencia.

\begin{tabular}{lll}
\hline & Experimentales & Obtenidos \\
\hline Energía de Enlace & $34.13 \mathrm{meV}$ & $31.50 \mathrm{meV}$ \\
Energía de FL & $(2.20-2.80) \mathrm{eV}$ & $(2.52-2.68) \mathrm{eV}$ \\
Longitud de Onda & $(440-560) \mathrm{nm}$ & $(440-540) \mathrm{nm}$
\end{tabular}

Tabla 1: Resumen comparativo de los datos de reportes experimentales y los obtenidos mediante un analisis teórico

del electrón y hueco, haciendo variar el espesor $L$ del pozo cuántico en el material de CdSe, logrando obtener una buena aproximación con los resultados experimentales.

Se resolvió numéricamente la ecuación de Schrödinger (ES) para obtener la energía mínima y de enlace del sistema (exciton), en el estudio de la (ES) independiente del tiempo se utilizó el Hamiltoniano en coordenadas cilíndricas del electrón-hueco, teniendo en cuenta solamente el exciton de hueco pesado. La (ES) fue resuelta por el método variacional y la aproximación de masa efectiva, para la heteroestructura de $\mathrm{ZnSe} / \mathrm{CdSe} / \mathrm{ZnSe}$ con valores de ancho de pozo en el intervalo $0.5 \mathrm{~nm} \leq \mathrm{L} \leq 70 \mathrm{~nm}$, la energía de enlace aquí calculada en relación a la obtenida en los experimentos presenta una buena aproximación que difiere en $2.63 \mathrm{meV}$. A partir de la gráfica de la energía de enlace figura (4) se determinó que el ancho crítico de brecha prohibido de la energía $E_{g}$ en el material del pozo cuántico (CdSe) tiene su energía de enlace máximo, cuando el ancho del pozo es de aproximadamente $3.8 \mathrm{~nm}$, valor que se encuentra en el límite del espesor critico sugerido por (D. Litvinovl, 2008a).

Los valores de la energía de emisión de la transición excitonica o energía de fotoluminiscencia (FL), para el estado base del exciton y la intensidad en función de la energía de transición, evidencia mediante las diferentes gráficas y de manera particular la gráfica de la longitud de onda en función de la energía de fotoluminiscencia figura (5b) que el espectro visible se encuentra en un rango del azul y verde con una tendencia mayor hacia el verde, con una pequeña variación comparado con los reportes experimentales 
(D. Litvinovl, 2008b)(I. Hernández-Calderón, 2005). Estos efectos de fotoluminiscencia pueden ser utilizados como referencia para el diseño de pozos cuánticos con emisión en la longitud de onda deseada, confinando los portadores de cargas para diferentes proporciones de altura del potencial y diferentes anchos de pozo. Los intervalos de longitudes de onda pueden ser tomadas como referencia para ciertos tipos de diodos laser (DL) y diodos emisores de luz (LED).

\section{REFERENCIAS}

A. Escamilla Esquivel, G. C. P. O. Z. A., J. Ortiz Lopez. (1997). Estudios ópticos de películas semiconductoras ternarias de $z n_{x} c d_{1-x} s e$ crecidas por csvt. , 43(5), 765-775.

A. Londoño Calderon, C. V. H. E. T. C. A. D., J. F. Jurado. (2011). Energía de exitones en pozos cuánticos ultra-delgados de znse/cdse/znse. , 43(5).

Chaudhuri, K. K. B. (1984). Effect of nonparabolicity on the energy levels of hydrogenic donors ingasasgalxalxas quamtum-well structures. , 29(4), 1803-1806.

Chuang, S. L. (1995). Physics of optoelectronic devices. New York.

D. Litvinovl, A. R. B. D. J. F. W. L. H. K. M. H., M.Schowalter. (2008a). Determination of critical thickness for defect formation of cdse/znse heterostructures by transmission electron microscopy and photoluminiscence spectroscopy. , 205(12), 2892-2897.

D. Litvinovl, A. R. B. D. J. F. W. L. H. K. M. H., M.Schowalter. (2008b). Determination of critical thickness for defect formation of cdse/znse heterostructures by transmission electron microscopy and photoluminiscence spectroscopy. , 205(12), 2892-2897.

Grundmann, M. (2010). The physics of semiconductors. second edition. (5).

I. Hernández-Calderón, A. A.-M. M.-R., J. C. Salcedo-Reyes. (2005). Finetuning of the emission of ultra-thin quantum wells of cdse and cdte by modication of the growth temperature. , 36(11), 985-988.

P. Aristizábal, W. A. O. C. A. D., R.L. Restrepo. (2007). Energía de enlace de excitones en pozos cuánticos de gaas/ga1xalxas. (7), 121-126.

P. Diaz Arencibia, I. H. C. J. M. A., O. de Melo Pereira. (2014). Analysis of optical transitions in $z n_{1-x} c d_{x} s e$ quamtum wells. Revista Mexicana de fisica Vol. 44 suplemento 3.

R. L. Greene, D. P. q. w. s., K. K. Bajaj. (1984). Energy levels of wannier exitons in gaasga1xalxas. , 29(4), 1807-1812.

Robert Eisberg, R. R. (2009). Física . Mexico DF.

T. Yao, J. C. (1998). Physics and applications of semiconductor quamtum structures.

V. Pellegrini, A. T. F. B. C. m. L. S. L. V. A. F., R. Atamasov. (1995). Excitonic properties of zn1xcdxse/znse strained quantum wells., 51(8), 5171-5175. 